Research Article

\title{
Complex Neutrosophic Soft Matrices Framework: An Application in Signal Processing
}

\author{
Madad Khan ${ }^{D},{ }^{1}$ Saima Anis, ${ }^{1}$ Kainat Bibi, ${ }^{1}$ Sohail Iqbal, ${ }^{2}$ and Florentin Smarandache ${ }^{3}$ \\ ${ }^{1}$ Department of Mathematics, COMSATS University Islamabad, Abbottabad Campus, Islamabad, Pakistan \\ ${ }^{2}$ Department of Mathematics, COMSATS University Islamabad, Islamabad Campus, Pakistan \\ ${ }^{3}$ Mathematics Department, University of New Mexico, 705 Gurley Ave., Gallup, NM 87301, USA \\ Correspondence should be addressed to Madad Khan; madadmath@yahoo.com
}

Received 29 September 2020; Revised 14 December 2020; Accepted 2 September 2021; Published 15 September 2021

Academic Editor: Efthymios G. Tsionas

Copyright $\odot 2021$ Madad Khan et al. This is an open access article distributed under the Creative Commons Attribution License, which permits unrestricted use, distribution, and reproduction in any medium, provided the original work is properly cited.

In this paper, we introduce the concept of complex neutrosophic soft matrices. We define some basic operations including complement, union, and intersection on these matrices. We extend the concept of complex neutrosophic soft sets to complex neutrosophic soft matrices and prove related properties. Moreover, we develop an algorithm using complex neutrosophic soft matrices and apply it in signal processing.

\section{Introduction}

The models of real-life problems in almost every field of science like mathematics, physics, operations research, medical sciences, engineering, computer science, artificial intelligence, and management sciences are mostly full of complexities. Many theories have been developed to overcome these uncertainties; one among those theories is fuzzy set theory. Zadeh was the first who gave the concept of a fuzzy set in 1965 [1]. Fuzzy sets are the generalizations or extensions of crisps sets.

In order to add the concept of nonmembership term to the definition of fuzzy set, the concept of an intuitionistic fuzzy set was introduced by Atanassov in 1986 [2], where he added the concept of nonmembership term to the definition of fuzzy set. The intuitionistic fuzzy set is characterized by a membership function $\mu$ and a nonmembership function $v$ with ranges $[0,1]$. The intuitionistic fuzzy set is the generalization of a fuzzy set. An intuitionistic fuzzy set can be applied in several fields including modeling, medical diagnosis, and decision-making. [3] Molodtsov introduced the concept of a soft set in 1999 and developed the fundamental results related to this theory. Basic operations including complement, union, and intersection are also defined on this set. Molodtsov used soft sets for applications in games, probability, and operational theories [3-6]. In 2018, Smarandache generalized the soft set to the hypersoft set by transforming the classical uniargument function $F$ into a multiargument function [7]. Maji et al. [8] introduced the concept of fuzzy soft sets by combining soft sets and fuzzy sets and applied them in decision-making problems [9]. In [10], Cagman and Enginolu used soft matrix theory for applications in decision-making problems.

The concept of neutrosophy was introduced by Smarandache [11] in 1998. A neutrosophic set is characterized by a truth membership function $T$, an indeterminacy function $I$, and a falsity membership function $F$. A neutrosophic set is a mathematical framework which generalizes the concept of a classical set, fuzzy set, intuitionistic fuzzy set, and interval valued fuzzy set [12]. In [13], Nabeeh introduced a method that can promote a personal selection process by integrating the neutrosophic analytical hierarchy process to show the proper solution among distinct options with order preference technique similar to an ideal solution (TOPSIS). In [14], Baset introduced a concept of a neutrosophy technique called type 2 neutrosophic numbers. By combining type 2 neutrosophic numbers and TOPSIS, they suggested a novel method T2NN-TOPSIS which has a lot of applications in group decision-making. They worked on a multicriteria group decision-making technique of the analytical network 
process method and Visekriterijusmska Optmzacija I Kommpromisno Resenje method under neutrosophic environment that deals high-order imprecision and incomplete information [15].

The largest number set is a complex set which is introduced by Gauss in 1795 and is the extension of a real number set. According to same fashion, a complex fuzzy set is extension to a fuzzy set as here the range set is extended from interval $[0,1]$ to a closed disc of radius one in complex plane. The degree of membership a complex fuzzy set is not restricted to a value in $[0,1]$; it is extended to a complex value lies in a disc of radius one in the complex plane.

Complex fuzzy sets are not simply a linear extension of conventional fuzzy sets; complex fuzzy sets allow a natural extension of fuzzy set theory to problems that are either very difficult or impossible to address with one-dimensional grades of membership. It is an obvious fact that uncertainty, indeterminacy, inconsistency, and incompleteness in data are periodic in nature. In order to address this difficulty, in 2002, Daniel Ramot was the first who gave the concept of a complex fuzzy set. The concept of a complex neutrosophic set was introduced in [16].

The complex fuzzy set $C$ is described as membership function, with range in closed unit disc in the complex plane. The complex-valued membership function $\phi_{s}(x)$ is defined as $\phi_{s}(x)=t_{s}(x) e^{i \cdot \eta_{s}(x)}$ that assigns a complex value of membership to any $x$ in $U$ (universal set) such that $t_{s}(x)$ and $\eta_{s}(x)$ both are real-valued with $t_{s}(x)$ is fuzzy set and $i=\sqrt{-1}$, where $t_{s}(x)$ is called amplitude term and $\eta_{s}(x)$ is called phase term.

Physically the complex fuzzy set is used for representing the complex fuzzy solar activity (solar maximum and solar minimum) through the measurement of sunspot number and is also used in signal processing. The complex neutrosophic set is the generalization of a complex fuzzy set and a neutrosophic set. The complex neutrosophic set is characterized by complex-valued truth membership function, complex-valued indeterminate function, and complex-valued falsehood function. In short, a complex neutrosophic set is more generalized because it is not only the generalization of all the current frameworks but also describes the information in a complete and comprehensive way.

A fuzzy set with its generalizations, like intuitionistic fuzzy sets, interval valued fuzzy sets, and cubic sets, represents uncertainties in models of the one-dimensional phenomenon while a complex fuzzy set is the only generalization of a fuzzy set which deals with the models of real-life problems with the two-dimensional and periodic phenomenon. A complex fuzzy set is more applicable because of its nature and can be used more widely in all branches of sciences. Since it is similar to that of a Fourier transform, more explicitly it is a particular sort of Fourier transform with the only restriction on the range which is a complex unit disc. A Fourier transform is used in signals and systems; that is, a Fourier transform is the mathematical tool for representing both continuous and discrete signals. Taking advantage of a complex fuzzy set, being a specific form of Fourier transform, it can be used to represent signals in a particular region of consideration. A neutrosophic set is the generalization of a fuzzy set which deals with the problems containing uncertainties of truthfulness, falsehood, and neutrality. The complex neutrosophic set has three major parts, that is, truth, intermediate, and falsehood membership functions. The truth membership function is totally the same as that of a complex fuzzy set while intermediate and falsehood membership functions are the new additions to it. Thus, a complex neutrosophic set can be applied more widely compared with other fuzzy sets.

In the vast area of science and technology, matrices play an important role. Classical matrix theory cannot solve all models of the daily life problems. In order to overcome these difficulties, Yang and Ji in [17] initiated a matrix representation of a fuzzy soft set and successfully applied the proposed notion of a fuzzy soft matrix in certain decisionmaking problems.

This work is basically the extension of the work of Ramot et al. [18], Alkouri and Saleh [19], Cai [20, 21], and Zhang et al. [22] to neutrosophic sets. Here, in this paper, we extend the concept by defining the complex neutrosophic fuzzy soft set and then the complex neutrosophic fuzzy soft matrix (CNFSM). Further, we discuss some basic operations on CNFSM and finally we develop an algorithm using these matrices and apply it in signal processing.

Soft matrices are widely used in signals and systems, decision-making problems, and medical diagnosis. This article has two aims. In the first part, we present theoretical foundations of the complex neutrosophic fuzzy soft matrices. These theoretical foundations provide basic notions and operations on complex neutrosophic soft matrices such as complex neutrosophic fuzzy soft zero matrix, complex neutrosophic fuzzy soft universal matrix, complex neutrosophic fuzzy soft submatrices, union of complex neutrosophic fuzzy soft matrices, intersection of complex neutrosophic fuzzy soft matrices, and complement of complex neutrosophic fuzzy soft matrices. Then, we introduce some fundamental results and discuss main strategies for applications of this concept in signals and systems, as well as a coherent discussion of the theory of complex neutrosophic fuzzy soft matrices. The aim of these new concepts is to provide a modern method with mathematical procedure to identify a reference signal out of large number of signals received by a digital receiver. The complex neutrosophic fuzzy soft matrix is the generalization of the fuzzy soft matrix, complex fuzzy soft matrix, and Pythagorean fuzzy soft matrix. The degree of membership function, nonmembership function, and phase terms are all applied to each entry of the matrix which give more fruitful results for a better choice in signals and systems along with other fields such as decision-making problems, medical diagnosis, and pattern recognition. These applied contexts provide solid evidence of the wide applications of the complex neutrosophic fuzzy soft matrices approach to signals and systems and decision-making problems.

\section{Preliminaries}

Here, we begin with a numerical example of a complex neutrosophic set which is already defined above. 
Example 1. Let $X=\left\{x_{1}, x_{2}, x_{3}\right\}$ be a universe of discourse.

Then, the complex neutrosophic set $S$ in $X$ is given as

$$
S=\frac{\left(0.6 e^{j 0.3}, e^{j \pi / 2}, 0.3 e^{j 0.6}\right)}{x_{1}}+\frac{\left(0.4 e^{j 0}, 0.9 e^{j \pi / 4}, 0.4 e^{j \pi / 4}\right)}{x_{2}}+\frac{\left(0.5 e^{j 2 \pi / 3}, 0.2 e^{j 0.2}, 0.7 e^{j \pi / 3}\right)}{x_{3}} .
$$

Definition 1 (fuzzy set (FS) [1]). Fuzzy set is defined by an arbitrary mapping from a nonempty set $X$ to the unit interval $[0,1]$, i.e., $f: X \longrightarrow[0,1]$. The set of all fuzzy subsets of $X$ is denoted by $F(X), \quad$ i.e., $\quad F(X)=\{f: f$ is a function from Xinto $[0,1]\}$.

Soft set theory is a generalization of fuzzy set theory, which was proposed by Molodtsov in 1999.

Definition 2 (soft set (SS) [3]). Let $U$ be the universal set, $E$ be the set of parameters, and $A \subseteq E$ and $P(U)$ be the power set of $U$, then a soft set $F_{A}$ is defined by a mapping.

$f_{A}: E \longrightarrow P(U)$ such that $f_{A}(x)=\phi$ if $x \notin A$.

In other words, we can say that soft set $F_{A}$ over $U$ is the parameterized family of subsets of $U$, that is, $F_{A}=\left\{\left(x, f_{A}(x)\right): x \in E, f_{A}(x) \in P(U)\right\}$.

Definition 3 (fuzzy soft set (FSS) [8]). Let $U$ be the universe of discourse, $E$ be the set of parameters, and $A \subseteq E$, then a fuzzy soft set $G_{A}$ is defined by a mapping: $g_{A}: E \longrightarrow P^{\prime}(U)$ where $P^{\prime}(U)$ is the collection of all fuzzy subsets of $U$, such that $g_{A}(x)=\phi$ if $x \notin A$.

In other words, we can say that fuzzy soft set $G_{A}$ over $U$ is the parameterized family of fuzzy subsets of $U$, that is, $G_{A}=\left\{\left(x, g_{A}(x)\right): x \in E, g_{A}(x) \in P^{\prime}(U)\right\}$.

Definition 4 (intuitionistic fuzzy set (IFS) [2]). An intuitionistic fuzzy set $I$ on a nonempty set $U$ (universal set) is defined by the set of triplets given by

$$
I=\left\{\left(x, \mu_{I}(x), \gamma_{I}(x)\right): x \in U\right\} .
$$

Here, $\mu_{I}(x)$ and $\gamma_{I}(x)$ both are functions from $U$ to $[0,1]$ as $\mu_{I}(x): U \longrightarrow[0,1]$ and $\gamma_{I}(x): U \longrightarrow[0,1]$. Here, $\mu_{I}(x)$ represents the degree of membership and $\gamma_{I}(x)$ represents the degree of nonmembership of each element $x \in U$ to the set $I$, respectively, also $0 \leq \mu_{I}(x)+\gamma_{I}(x) \leq 2$, for all $x \in U$.

Definition 5 (complex fuzzy set (CFS) [18]). The complex fuzzy set $S$ on universe of discourse $X$ is described as complex-valued membership function $\mu_{S}(x)$ that assigns value of membership of the form $r_{s}(x) e^{j w_{s}(x)}$ to any element $x \in X$, where $j=\sqrt{-1}, \mu_{S}(x)$ involves two real-valued $r_{s}(x)$ and $w_{s}(x)$, with $r_{s}(x) \in[0,1]$.
Mathematically, $S=\left\{\left(x, \mu_{s}(x)\right): x \in X\right\}$.

Definition 6 (complex intuitionistic fuzzy set (CIFS) [19]). The complex intuitionistic fuzzy set $C I$ on a nonempty set $U$ (universal set) is defined by the set of triplets given by $C I=\left\{\left(x, \mu_{C I}(x), \gamma_{C I}(x)\right): x \in U\right\} . \quad$ Here, $\quad \mu_{C I}(x)=$ $r_{C I}(x) e^{j \omega_{C I}(x)}$ and $\gamma_{C I}(x)=l_{C I}(x) e^{j m_{C I}(x)}$ both are functions from $U$ to closed unit disc in the complex plane and also $\mu_{C I}(x)$ represents the degree of membership and $\gamma_{C I}(x)$ represents the degree of nonmembership of each element $x \in U$ to the set $C I$, respectively, and also $0 \leq r_{C I}(x)+l_{C I}(x) \leq 2$, for all $x \in U$.

Definition 7 (complex neutrosophic fuzzy set (CNFS) [16]). The complex neutrosophicfuzzy set $N$ on a nonempty set $U$ (universal set) is defined by the set as $N=\{(x$, $\left.\left.T_{N}(x), I_{N}(x), F_{N}(x): x \in U\right)\right\} . \quad$ Here, $\quad T_{N}(x)=r_{N}(x)$ $e^{j \omega_{N}(x)}, I_{N}(x)=l_{N}(x) e^{j m_{N}(x)}$, and $F_{N}(x)=p_{N}(x) e^{j q_{N}(x)}$ are the complex-valued functions from $U$ to the closed unit disc in the complex plane where $T_{N}(x)$ describes complexvalued truth membership function, $I_{N}(x)$ describes complex-valued indeterminate membership function, and $F_{N}(x)$ describes complex-valued falsehood membership function of each element $x \in U$ to the set $N$, respectively, and also $0 \leq r_{N}(x)+l_{N}(x)+p_{N}(x) \leq 3$, for all $x \in U$.

\section{Complex Neutrosophic Fuzzy Soft Matrix Theory}

In this section, we introduced a new concept of complex neutrosophic fuzzy soft matrices. We defined the operations of union, intersection, compliment, and submatrices. We defined zero and universal matrices. Moreover, we proved some related results.

Definition 8 (complex neutrosophic fuzzy soft matrix (CNFSM)). Consider a universal set $U=\left\{u_{1}, u_{2}, u_{3}, \ldots, u_{m}\right\}$ and set of parameters $E=\left\{e_{1}, e_{2}, e_{3}, \ldots, e_{n}\right\}$ such that $A \subseteq E$ and $\left(c_{A}, A\right)$ be a complex neutrosophic fuzzy soft set over $(U, E)$. Then, the CNFSS $\left(c_{A}, A\right)$ in matrix form is represented by $A_{m \times n}=\left[a_{i j}\right]_{m \times n}$ or $A=\left[a_{i j}\right] \quad$ where $i=1,2,3, \ldots, m$ and $j=1,2,3, \ldots, n$.

$$
\text { Here, } a_{i j}= \begin{cases}\left|\mu_{j}\left(u_{i}\right)\right|=\left(\left|\mu_{j}^{T}\left(u_{i}\right)\right|,\left|\mu_{j}^{I}\left(u_{i}\right)\right|,\left|\mu_{j}^{F}\left(u_{i}\right)\right|\right), & \text { if } e_{j} \in A, \\ (0,0,0) & \text { if } e_{j} \notin A .\end{cases}
$$


Now, $\left(\mu_{j}^{T}\left(u_{i}\right), \mu_{j}^{I}\left(u_{i}\right), \mu_{j}^{F}\left(u_{i}\right)\right)$ represents degrees of membership of truth, intermediate, and falsehood on $u_{i}$. Throughout this paper, we will use the abbreviation CNFSM $_{m \times n}$ for complex neutrosophic fuzzy soft matrix over $U$. Following is the example of a complex neutrosophic fuzzy soft matrix.
Example 2. Let $U=\left\{u_{1}, u_{2}, u_{3}\right\}$ be a universal set representing the three firms, $E=\left\{e_{1}\right.$ (costly), $e_{2}$ (beautiful), $e_{3}$ (luxurious) $\}$ be the parameters set, and $A=\left\{e_{1}, e_{2}\right\} \subseteq E$. Then, CNFSS $\left(c_{A}, A\right)$ over the universal set $U$ is given by

$$
\begin{aligned}
\left(c_{A}, A\right) & =\left\{c_{A}\left(e_{1}\right)=\left\{\left(u_{1},\left(\left|0.3 e^{j \pi}\right|,\left|0.6 e^{j \pi / 2}\right|,\left|e^{j \pi}\right|\right)\right)\right\},\left(u_{2},\left(\left|0.7 e^{j \pi / 4}\right|,\left|0.8 e^{j \pi / 4}\right|,\left|0.5 e^{j \pi / 2}\right|\right)\right),\left(u_{3},\left(\left|0.9 e^{j \pi}\right|,\left|0.1 e^{j \pi / 6}\right|,\left|0.2 e^{j \pi / 2}\right|\right)\right), c_{A}\left(e_{2}\right)\right. \\
& \left.=\left\{\left(u_{1},\left(\left|0.1 e^{j \pi / 3}\right|,\left|0.2 e^{j \pi / 6}\right|,\left|0.1 e^{j \pi}\right|\right)\right),\left(u_{2},\left(\left|0.3 e^{j \pi / 2}\right|,\left|0.9 e^{j \pi / 2}\right|,\left|0.9 e^{j \pi / 4}\right|\right)\right),\left(u_{3},\left(\left|0.5 e^{j \pi / 3}\right|,\left|0.5 e^{j \pi}\right|,\left|0.6 e^{j \pi / 3}\right|\right)\right)\right\}\right\} .
\end{aligned}
$$

Here,

$$
\begin{aligned}
& 0.3 e^{j \pi}=0.3(\cos \pi+j \sin \pi)=0.3(-1+0)=-0.3 \\
& \left|0.3 e^{j \pi}\right|=|-0.3|=0.30 .6 \\
& 6 e^{j \pi / 2}=0.6\left(\cos \left(\frac{\pi}{2}\right)+j \sin \left(\frac{\pi}{2}\right)\right)=0.6(0+j)=0.6 j \\
& \left|0.6 e^{j \pi / 2}\right|=|0.6 j|=\sqrt{0.36}=0.6 \\
& e^{j \pi}=\cos \pi+j \sin \pi=-1+0=-1 \\
& \left|e^{j \pi}\right|=|-1|=1,0.7 e^{j \pi / 4}=0.7\left(\cos \left(\frac{\pi}{4}\right)+j \sin \left(\frac{\pi}{4}\right)\right)=0.7\left(\frac{1}{\sqrt{2}}+j \frac{1}{\sqrt{2}}\right) \\
& =0.7(0.707+j 0.707)=0.494+j 0.494 \\
& \left|0.7 e^{j \pi / 4}\right|=|0.494+j 0.494|=\sqrt{0.244+0.244}=0.69, \\
& 0.8 e^{j \pi / 4}=0.8\left(\cos \left(\frac{\pi}{4}\right)+j \sin \left(\frac{\pi}{4}\right)\right)=0.8\left(\frac{1}{\sqrt{2}}+j \frac{1}{\sqrt{2}}\right) \\
& =0.8(0.707+j 0.707)=0.5656+j 0.5656 \text {, } \\
& \left|0.8 e^{j \pi / 4}\right|=|0.5656+j 0.5656|=\sqrt{0.319+0.319}=0.790 .5 \\
& 0.5 e^{j \pi / 2}=0.5\left(\cos \left(\frac{\pi}{2}\right)+j \sin \left(\frac{\pi}{2}\right)\right)=0.5 j \\
& \left|0.5 e^{j \pi / 2}\right|=|0.5 j|=\sqrt{0.25}=0.5 \\
& 0.9 e^{j \pi}=0.9(\cos \pi+j \sin \pi)=0.9(-1)=-0.9 \\
& \left|0.9 e^{j \pi}\right|=|-0.9|=0.9 \\
& 0.1 e^{j \pi / 6}=0.1\left(\cos \left(\frac{\pi}{6}\right)+j \sin \left(\frac{\pi}{6}\right)\right)=0.1(0.866+j 0.5)=0.0866+j 0.05 \\
& \left|0.1 e^{j \pi / 6}\right|=|0.0866+j 0.05|=\sqrt{0.0074+0.0025}=0.099,
\end{aligned}
$$




$$
\begin{aligned}
& 0.2 e^{j \pi / 2}=0.2\left(\cos \left(\frac{\pi}{2}\right)+j \sin \left(\frac{\pi}{2}\right)\right)=0.2(0+j)=0.2 j \\
& \left|0.2 e^{j \pi / 2}\right|=|0.2 j|=\sqrt{0.04}=0.2 \text {, } \\
& 0.1 e^{j \pi / 3}=0.1\left(\cos \left(\frac{\pi}{3}\right)+j \sin \left(\frac{\pi}{3}\right)\right)=0.1(0.5+j 0.866)=0.05+j 0.0866 \\
& \left|0.1 e^{j \pi / 3}\right|=|0.05+j 0.0866|=\sqrt{0.0025+0.0074}=0.090 .2 e^{j \pi / 6} \\
& =0.2\left(\cos \left(\frac{\pi}{6}\right)+j \sin \left(\frac{\pi}{6}\right)\right)=0.2(0.866+j 0.5)=0.1732+j 0.1 \\
& \left|0.2 e^{j \pi / 6}\right|=|0.1732+j 0.1|=\sqrt{0.029+0.01}=0.19 \text {, } \\
& 0.1 e^{j \pi}=0.1(\cos \pi+j \sin \pi)=0.1(-1+0)=-0.1 \\
& \left|0.1 e^{j \pi}\right|=|-0.1|=0.1 \\
& 0.3 e^{j \pi / 2}=0.3\left(\cos \left(\frac{\pi}{2}\right)+j \sin \left(\frac{\pi}{2}\right)\right)=0.3 j \\
& \left|0.3 e^{j \pi / 2}\right|=|0.3 j|=\sqrt{0.09}=0.3 \\
& 0.9 e^{j \pi / 2}=0.9\left(\cos \pi / 2+j \sin \left(\frac{\pi}{2}\right)\right)=0.9 j \\
& \left|0.9 e^{j \pi / 2}\right|=|0.9 j|=\sqrt{0.81}=0.9 \text {, } \\
& 0.9 e^{j \pi / 4}=0.9\left(\cos \left(\frac{\pi}{4}\right)+j \sin \left(\frac{\pi}{4}\right)\right)=0.9\left(\frac{1}{\sqrt{2}}+j \frac{1}{\sqrt{2}}\right) \\
& =0.9(0.707+j 0.707)=0.636+j 0.636 \\
& \left|0.9 e^{j \pi / 4}\right|=|0.636+j 0.636|=\sqrt{0.404+0.404}=0.898 \text {, } \\
& 0.5 e^{j \pi / 3}=0.5\left(\cos \left(\frac{\pi}{3}\right)+j \sin \left(\frac{\pi}{3}\right)\right)=0.5(0.5+j 0.866)=0.25+j 0.433 \\
& \left|0.5 e^{j \pi / 3}\right|=|0.25+j 0.433|=\sqrt{0.0625+0.187}=0.499 \\
& 0.5 e^{j \pi}=0.5(\cos \pi+j \sin \pi)=0.5(-1)=-0.5 \\
& \left|0.5 e^{j \pi}\right|=|-0.5|=0.5 \\
& 0.6 e^{j \pi / 3}=0.6\left(\cos \left(\frac{\pi}{3}\right)+j \sin \left(\frac{\pi}{3}\right)\right)=0.6(0.5+j 0.866)=0.3+j 0.519 \\
& \left|0.6 e^{j \pi / 3}\right|=|0.3+j 0.519|=\sqrt{0.09+0.269}=0.599 \text {. }
\end{aligned}
$$

Now, the abovementioned CNFSS $\left(c_{A}, A\right)$ in matrix form is given by

$$
A=\left[\begin{array}{ccc}
(0.3,0.6,1) & (0.09,0.19,0.1) & (0,0,0) \\
(0.69,0.79,0.5) & (0.3,0.9,0.898) & (0,0,0) \\
(0.9,0.099,0.2) & (0.499,0.5,0.599) & (0,0,0)
\end{array}\right]
$$

Definition 9 (complex neutrosophic fuzzy soft zero natrix). Let $\left[a_{i j}\right] \in C N F S M_{m \times n}$, then $\left[a_{i j}\right]$ is called complex neutrosophic fuzzy soft zero matrix if $\left(a_{i j}, r_{i j}, l_{i j}\right)=(0,0,0)$, for all $i$ and $j$, and is denoted by [0].

\section{Example 3}

$$
[0]=\left[\begin{array}{lll}
(0,0,0) & (0,0,0) & (0,0,0) \\
(0,0,0) & (0,0,0) & (0,0,0) \\
(0,0,0) & (0,0,0) & (0,0,0)
\end{array}\right]
$$

Definition 10 (complex neutrosophic fuzzy soft universal matrix). Let $\left[a_{i j}\right] \in \mathrm{CNFSM}_{m \times n}$, then $\left[a_{i j}\right]$ is called complex neutrosophic fuzzy soft universal matrix if $\left(a_{i j}, r_{i j}, l_{i j}\right)=(1,1,1)$, for all $i$ and $j$, and is represented by [1]. 


$$
[1]=\left[\begin{array}{lll}
(1,1,1) & (1,1,1) & (1,1,1) \\
(1,1,1) & (1,1,1) & (1,1,1) \\
(1,1,1) & (1,1,1) & (1,1,1)
\end{array}\right]
$$

Definition 11 (complex neutrosophic fuzzy soft submatrices). Let $A_{m \times n}$ and $B_{m \times n}$ be two CNFSMs, then

(i) $A_{m \times n}$ is a CNFS submatrix of $B_{m \times n}$ and is denoted by $A_{m \times n} \sqsubseteq B_{m \times n} \quad$ if $\quad a_{i j}=\left(a_{i j}, a_{i j}^{\prime}, a_{i j}^{\prime \prime}\right) \preccurlyeq b_{i j}=\left(b_{i j}\right.$, $\left.b_{i j}^{\prime}, b_{i j}^{\prime \prime}\right)$, that is, $\left(a_{i j} \preccurlyeq b_{i j}, a_{i j}^{\prime \prime} \preccurlyeq b_{i j}^{\prime}, a_{i j}^{\prime \prime} \preccurlyeq b_{i j}^{\prime \prime}\right)$, for all $a_{i j} \in A_{m \times n}, b_{i j} \in B_{m \times n}$

(ii) $A_{m \times n}$ is a proper CNFS submatrix of $B_{m \times n}$ and is denoted by $A_{m \times n} \sqsubset B_{m \times n}$ if $a_{i j}=\left(a_{i j}, a_{i j}^{\prime}, a_{i j}^{\prime \prime}\right) \prec b_{i j}=$ $\left(b_{i j}, b_{i j}^{\prime}, b_{i j}^{\prime \prime}\right)$, that is, $\left(a_{i j}<b_{i j}, a_{i j}^{\prime} \prec b_{i j}^{\prime}, a_{i j}^{\prime \prime}<b_{i j}^{\prime \prime}\right)$, for all $a_{i j} \in A_{m \times n}, b_{i j} \in B_{m \times n}$ and for at least one entry $a_{i j} \prec b_{i j}$, that is, $\left(a_{i j} \prec b_{i j}, a_{i j}^{\prime} \prec b_{i j}^{\prime}, a_{i j}^{\prime \prime} \prec b_{i j}^{\prime \prime}\right)$

(iii) Two CNFSMs $A_{m \times n}$ and $B_{m \times m n}$ are equal and are denoted by $A_{m \times n}=B_{m \times n}$, if $a_{i j}=\left(a_{i j}, a_{i j}^{\prime}, a_{i j}^{\prime \prime}\right)=$ $b_{i j}=\left(b_{i j}, b_{i j}^{\prime}, b_{i j}^{\prime \prime}\right)$, that is. $\left(a_{i j}=b_{i j}, a_{i j}^{\prime}=\right.$ $\left.b_{i j}^{\prime}, a_{i j}^{\prime \prime}=b_{i j}^{\prime \prime}\right)$, for all $a_{i j} \in A_{m \times n}, b_{i j} \in B_{m \times n}$

Example 4. Let

$$
\begin{aligned}
& A_{2 \times 2}=\left[\begin{array}{ll}
(0.2,0.4,0.1) & (0.1,0.5,0.2) \\
(0.3,0.7,0.3) & (0.5,0.4,0.4)
\end{array}\right], \\
& B_{2 \times 2}=\left[\begin{array}{ll}
(0.2,0.4,0.1) & (0.3,0.7,0.9) \\
(0.3,0.7,0.3) & (0.7,0.5,0.7)
\end{array}\right] .
\end{aligned}
$$

So, we can write that $A_{2 \times 2} \sqsubset B_{2 \times 2}$. Moreover, $A \sqsubset B$.

Definition 12. (union/intersection and compliment of complex neutrosophic fuzzy soft matrices).

Let $A_{m \times n}$ and $B_{m \times n}$ be two CNFSM, then the CNFSM $C_{m \times n}$ is called

(i) Union of $A_{m \times n}$ and $B_{m \times n}$ and is denoted by $A_{m \times n} \sqcup B_{m \times n}$ if $C_{m \times n}=\max \left\{A_{m \times n}, B_{m \times n}\right\}$, for all $i$ and $j$, that is, $c_{i j}=\left(\max \left(a_{i j}, b_{i j}\right), \min \left(a_{i j}^{\prime}, b_{i j}^{\prime}\right)\right.$, $\left.\min \left(a_{i j}^{\prime \prime}, b_{i j}^{\prime \prime}\right)\right)$ where $c_{i j}=\left(c_{i j}, c_{i j}, c_{i j}^{\prime \prime}\right)$

(ii) Intersection of $A_{m \times n}$ and $B_{m \times n}$ is denoted by $A_{m \times n} \sqcap B_{m \times n}$ if $C_{m \times n}=\min \left\{A_{m \times n}, B_{m \times n}\right\}$, for all $i$ and $j$, that is, $c_{i j}=\left(\min \left(a_{i j}, b_{i j}\right), \max \left(a_{i j}^{\prime}, b_{i j}^{\prime}\right)\right.$, $\left.\max \left(a_{i j}^{\prime \prime}, b_{i j}^{\prime \prime}\right)\right)$, where $c_{i j}=\left(c_{i j}, c_{i j}^{\prime}, c_{i j}^{\prime \prime}\right)$

(iii) Complement of $A_{m \times n}$ is denoted by $A_{m \times n}^{\prime}$ if $C_{m \times n}=1-A_{m \times n}$, for all $i$ and $j$, that is, $c_{i j}=\left(1-a_{i j}, 1-a_{i j}^{\prime}, 1-a_{i j}^{\prime \prime}\right)$, where $\quad c_{i j}=\left(c_{i j}\right.$, $\left.c_{i j}^{\prime}, c_{i j}^{\prime \prime}\right)$

Example 5. Assume that

$$
\begin{aligned}
& A_{2 \times 2}=\left[\begin{array}{cc}
(0.3,0.6,1) & (0.65,0,0.6) \\
(0.3,0.9,0) & (0.8,0.7,0.9)
\end{array}\right], \\
& B_{2 \times 2}=\left[\begin{array}{cc}
(0.49,0.5,0.4) & (0.2,0,0.3) \\
(0.1,0.9,0.3) & (0,0,0)
\end{array}\right],
\end{aligned}
$$

then,

$$
\begin{aligned}
A_{2 \times 2} \sqcup B_{2 \times 2} & =\left[\begin{array}{cc}
(0.49,0.5,0.4) & (0.65,0,0.3) \\
(0.3,0.9,0) & (0.8,0,0)
\end{array}\right], \\
A_{2 \times 2} \sqcap B_{2 \times 2} & =\left[\begin{array}{cc}
(0.3,0.6,1) & (0.2,0,0.6) \\
(0.1,0.9,0.3) & (0,0.7,0.9)
\end{array}\right], \\
A_{2 \times 2}^{\prime} & =\left[\begin{array}{cc}
(0.7,0.4,0) & (0.35,1,0.4) \\
(0.7,0.1,1) & (0.2,0.3,0.1)
\end{array}\right] .
\end{aligned}
$$

Proposition 1. Let $A_{m \times n}$ be a CNFSM, then

$$
\begin{aligned}
& \text { (i) }\left(\left(A_{m \times n}\right)^{\prime}\right)^{\prime}=A_{m \times n} \text {, } \\
& \text { (ii) }[0]^{\prime}=[1] .
\end{aligned}
$$

Proof. It follows from definition.

Proposition 2. Let $A_{m \times n}, B_{m \times n}$, and $C_{m \times n}$ be three CNFSMs, then

$$
\begin{aligned}
& \text { (i) } A_{m \times n}=B_{m \times n} \text { and } B_{m \times n}=C_{m \times n} \Longrightarrow A_{m \times n}=C_{m \times n} \text {, } \\
& \text { (ii) } A_{m \times n} \sqsubseteq B_{m \times n} \text { and } B_{m \times n} \sqsubseteq A_{m \times n} \Longrightarrow A_{m \times n}=B_{m \times n} \text {. }
\end{aligned}
$$

Proof. It follows from definition.

Proposition 3. Let $A_{m \times n}$ and $B_{m \times n}$ be two CNFSMs, then

$$
A_{m \times n} \sqsubseteq B_{m \times n} \text { and } B_{m \times n} \sqsubseteq C_{m \times n} \Longrightarrow A_{m \times n} \sqsubseteq C_{m \times n} .
$$

Proof. It follows from definition.

Proposition 4. Let $A_{m \times n}$ and $B_{m \times n}$ be two CNFSMs, then

(i) $A_{m \times n} \sqcup B_{m \times n}=B_{m \times n} \sqcup A_{m \times n}$,

(ii) $A_{m \times n} \sqcap B_{m \times n}=B_{m \times n} \sqcap A_{m \times n}$,

(iii) $\left(A_{m \times n} \sqcup B_{m \times n}\right) \sqcup C_{m \times n}=A_{m \times n} \sqcup\left(B_{m \times n} \sqcup C_{m \times n}\right)$,

(iv) $\left(A_{m \times n} \sqcap B_{m \times n}\right) \sqcap C_{m \times n}=A_{m \times n} \sqcap\left(B_{m \times n} \sqcap C_{m \times n}\right)$,

(v) $A_{m \times n} \sqcup\left(B_{m \times n} \sqcap C_{m \times n}\right)=\left(A_{m \times n} \sqcup B_{m \times n}\right) \sqcap\left(A_{m \times n} \sqcup C_{m \times n}\right)$,

(vi) $A \sqcap\left(B_{m \times n} \sqcup C_{m \times n}\right)=\left(A_{m \times n} \sqcap B_{m \times n}\right) \sqcup\left(A_{m \times n} \sqcap C_{m \times n}\right)$. 
Proof

$$
\begin{aligned}
\text { (i) } A_{m \times n} \sqcup B_{m \times n} & =\max \left(A_{m \times n}, B_{m \times n}\right) \\
& =\max \left(B_{m \times n}, A_{m \times n}\right) \\
& =B_{m \times n} \sqcup A_{m \times n}, \\
\text { (ii) } A_{m \times n} \sqcap B_{m \times n} & =\min \left(A_{m \times n}, B_{m \times n}\right) \\
& =\min \left(B_{m \times n}, A_{m \times n}\right) \\
& =B_{m \times n} \sqcap A_{m \times n}, \\
\text { (iii) }\left(A_{m \times n} \sqcup B_{m \times n}\right) \sqcup C_{m \times n} & =\max \left(\left(A_{m \times n} \sqcup B_{m \times n}\right), C_{m \times n}\right) \\
& =\max \left(\max \left(A_{m \times n}, B_{m \times n}\right), C_{m \times n}\right) \\
& =\max \left(A_{m \times n}, \max \left(B_{m \times n}, C_{m \times n}\right)\right) \\
& =\max \left(A_{m \times n},\left(B_{m \times n} \sqcup C_{m \times n}\right)\right) \\
& =A_{m \times n} \sqcup\left(B_{m \times n} \sqcup C_{m \times n}\right), \\
\text { (iv) }\left(A_{m \times n} \sqcap B_{m \times n}\right) \sqcap C_{m \times n} & =\min \left(\left(A_{m \times n} \sqcap B_{m \times n}\right), C_{m \times n}\right) \\
& =\min \left(\min \left(A_{m \times n}, B_{m \times n}\right), C_{m \times n}\right) \\
& =\min \left(A_{m \times n}, \min \left(B_{m \times n}, C_{m \times n}\right)\right) \\
& =\min \left(A_{m \times n},\left(B_{m \times n} \sqcap C_{m \times n}\right)\right) \\
& =A_{m \times n} \sqcap\left(B_{m \times n} \sqcap C_{m \times n}\right) . \\
\text { (vi) } A_{m \times n} \sqcap\left(B_{m \times n} \sqcup C_{m \times n}\right) & =\min \left(A_{m \times n},\left(B_{m \times n} \sqcup C_{m \times n}\right)\right) \\
& =\min \left(A_{m \times n}, \max \left(B_{m \times n}, C_{m \times n}\right)\right) \\
& =\max \left(\min \left(A_{m \times n}, B_{m \times n}\right), \min \left(A_{m \times n}, C_{m \times n}\right)\right) \\
& =\max \left(\left(A_{m \times n} \sqcap B_{m \times n}\right),\left(A_{m \times n} \sqcap C_{m \times n}\right)\right) \\
& =\left(A_{m \times n} \sqcap B_{m \times n}\right) \sqcup\left(A_{m \times n} \sqcap C_{m \times n}\right) . \\
\text { (v) } A_{m \times n} \sqcup\left(B_{m \times n} \sqcap C_{m \times n}\right) & =\max \left(A_{m \times n},\left(B_{m \times n} \sqcap C_{m \times n}\right)\right) \\
& =\max \left(A_{m \times n}, \min \left(B_{m \times n}, C_{m \times n}\right)\right) \\
& =\min \left(\max \left(A_{m \times n}, B_{m \times n}\right), \max \left(A_{m \times n}, C_{m \times n}\right)\right) \\
& =\min \left(\left(A_{m \times n} \sqcup B_{m \times n}\right),\left(A_{m \times n} \sqcup C_{m \times n}\right)\right) \\
& \left(A_{m \times n} \sqcup B_{m \times n}\right) \sqcap\left(A_{m \times n} \sqcup C_{m \times n}\right), \\
& \\
&
\end{aligned}
$$

Proposition 5. Let $A_{m \times n}$ and $B_{m \times n}$ be two CNFSMs, then the De-Morgan laws are valid:

$$
\begin{aligned}
& \text { (i) }\left(A_{m \times n} \sqcup B_{m \times n}\right)^{\prime}=\left(A_{m \times n}\right)^{\prime} \sqcap\left(B_{m \times n}\right)^{\prime} \\
& \text { (ii) }\left(A_{m \times n} \sqcap B_{m \times n}\right)^{\prime}=\left(A_{m \times n}\right)^{\prime} \sqcup\left(B_{m \times n}\right)^{\prime} .
\end{aligned}
$$

Proof.

$$
\text { (i) } \begin{aligned}
\left(A_{m \times n} \sqcup B_{m \times n}\right)^{\prime} & =\left[\max \left(A_{m \times n}, B_{m \times n}\right)\right]^{\prime} \\
& =\left[1-\max \left(A_{m \times n}, B_{m \times n}\right)\right] \\
& =\left[\min \left(1-A_{m \times n}, 1-B_{m \times n}\right)\right] \\
& =\left[A_{m \times n}\right]^{\prime} \sqcap\left[B_{m \times n}\right]^{\prime}, \\
\left(A_{m \times n} \sqcap B_{m \times n}\right)^{\prime} & =\left[\min \left(A_{m \times n}, B_{m \times n}\right)\right]^{\prime} \\
& =\left[1-\min \left(A_{m \times n}, B_{m \times n}\right)\right] \\
& =\left[\max \left(1-A_{m \times n}, 1-B_{m \times n}\right)\right] \\
& =\left[A_{m \times n}\right]^{\prime} \sqcup\left[B_{m \times n}\right]^{\prime} .
\end{aligned}
$$

\section{Complex Neutrosophic Fuzzy Soft Decision- Making Method}

Now, we are going to discuss real-life applications of newly defined $\mathrm{CNFSM}_{m \times n}$. We will show how our theoretical concepts and results can be applied to the real-life phenomenon. Specifically, we will show that $\mathrm{CNFSM}_{m \times n}$ explains how to get a better and clear signal for identification with a given reference signal. Before moving towards the algorithm, we will define the fuzzy soft (FS) max-min decision-making method (FSMmDM) by using FS max-min decision function and also define here the optimum FS on universal set $U$.

Definition 13 (fuzzy soft (FS) max-min decision-making function [10]). Let $\left[c_{i p}\right] \in S M_{m \times n^{2}}, \quad I_{k}=\{p$ : thereexisti, $\left.c_{i p} \neq 0,(k-1) n<p \leq k n\right\}$, for all $k \in I=\{1,2,3, \ldots, n\}$. Then, soft max-min decision function, denoted $M m$, is defined as follows:

$$
M m: S M_{m \times n^{2}} \longrightarrow S M m_{m \times 1}, M m\left[c_{i p}\right]=\left[\max _{k \in I}\left\{t_{k}\right\}\right],
$$

where

$$
t_{k}=\left(\begin{array}{cc}
\min _{p \in I_{k}}\left\{c_{i p}\right\}, & \text { if } I_{k} \neq\{\}, \\
0, & \text { if } I_{k}=\{\} .
\end{array}\right)
$$

The one column soft matrix $M m\left[c_{i p}\right]$ is called max-min soft decision-making matrix.

Definition 14 (see [10]). Let $U=\left\{u_{1}, u_{2}, \ldots, u_{m}\right\}$ be a universal set and $M m\left[c_{i p}\right]=\left[d_{i 1}\right]$. Then, a subset of $U$ can be obtained by using $\left[d_{i 1}\right]$ as in the following way $\operatorname{opt}_{\left[d_{i 1}\right]}(U)=\left\{u_{i}: u_{i} \in U, d_{i 1}=1\right\}$, which is called an optimum set on $U$.

\subsection{Decision-Making Algorithm}

step 1. Suppose that $\mathbf{M}$ different signals $S_{1}\left(t^{\prime}\right)$, $S_{2}\left(t^{\prime}\right), \ldots, S_{M}\left(t^{\prime}\right)$ are detected and sampled by a receiver and let $U=\left\{S_{1}\left(t^{\prime}\right), S_{2}\left(t^{\prime}\right), \ldots, S_{M}\left(t^{\prime}\right)\right\}$. Each of these signals is sampled $N$ times. Let $S_{m}\left(r^{\prime}\right)$ denote the $r^{\prime}$ th sample $\left(1 \leq r^{\prime} \leq N\right)$ of the $m$ th signal $(1 \leq m \leq M)$. Now, we know that each signal has its Fourier transform. So, each received signal can be expressed as summation of its Fourier components as

$$
\begin{gathered}
S_{m}\left(r^{\prime}\right)=\left(\frac{1}{N}\right) \sum_{n=1}^{N} C_{m, n} e^{i 2 \pi(n-1)\left(r^{\prime}-1\right) / N}, \text { then } \\
\left|S_{m}\left(r^{\prime}\right)\right|=\left(\frac{1}{N}\right) \sum_{n=1}^{N}\left|C_{m, n}\right| \cdot\left|e^{i 2 \pi(n-1)\left(r^{\prime}-1\right) / N}\right|,
\end{gathered}
$$

where $C_{m, n}(1 \leq n \leq N)$ represents complex Fourier coefficients of $S_{m}$. The above expression can also be rewritten as follows: 
$\left|S_{m}\left(r^{\prime}\right)\right|=(1 / N) \sum_{n=1}^{N}\left|B_{m, n}\right| \cdot\left|e^{i\left(2 \pi(n-1)\left(r^{\prime}-1\right)+N \beta_{m, n}\right) / N}\right|$, where $C_{m, n}=B_{m, n} e^{i \beta_{m, n}}$, with $B_{m, n}, \beta_{m, n}$ real-valued and $B_{m, n} \geq 0$, for all $n$, where $1 \leq n \leq N$. step 2. The above given signals are expressed as in matrix form as $A=\left[\left|S_{m}\left(r^{\prime}\right)\right|\right]_{N \times M}$, that is, express $N$ samples of each signal (total $M$ signals) in columns:

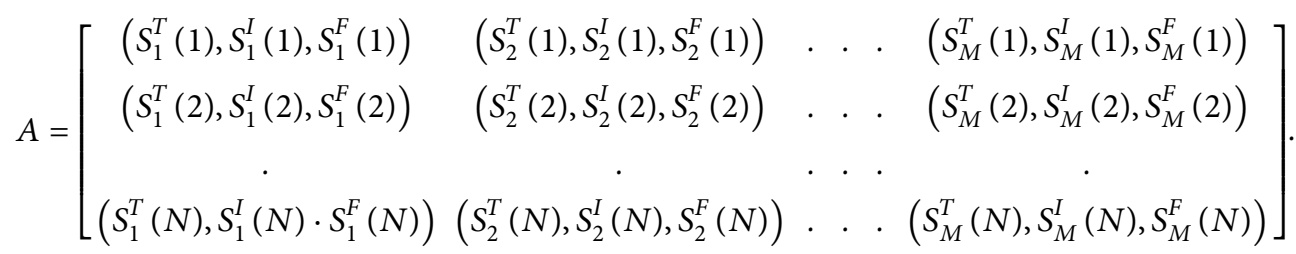

step 3. Similarly, we will construct another matrix by the signals $S_{m}^{*}(r)$.

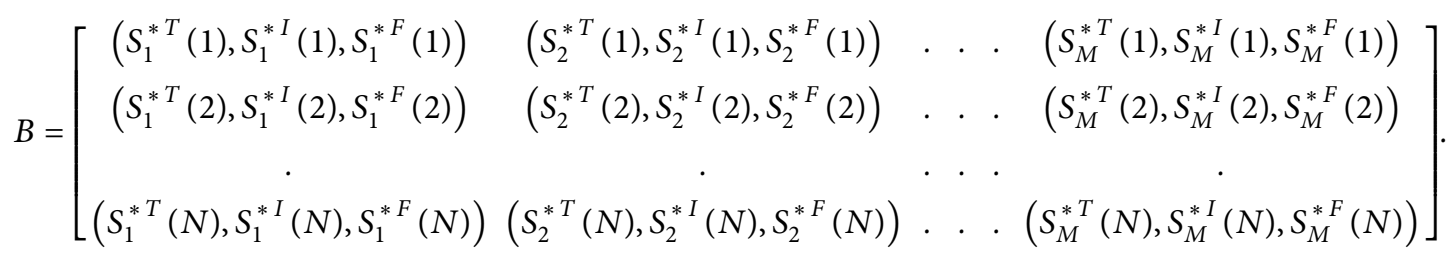

step 4. Multiply matrices $A$ and $B$ using usual multiplication of matrices. In this multiplication, the truth value of the entry of the first matrix will be multiplied by the truth value of the entry of the second matrix. The intermediate and false values of the entries are multiplied similarly. step 5. The complex neutrosophic fuzzy soft max-min decision-making matrix (CNFSMmDM) is found by taking minimum of truth, intermediate memberships, and maximum of falsehood membership values of each column, and we will get a column matrix $\left[d_{i 1}\right]$, where $1 \leq i \leq M$.

step 6. An optimum set opt $\operatorname{Mm}_{M[A B]}(U)$ on $U$ is found, that is,

$$
\left\{\left(\max \left\{\left|S_{j}^{T}(i)\right|, \max \left\{\left|S_{j}^{I}\left(u_{i}\right)\right|\right\}, \min \left|S_{j}^{F}\left(u_{i}\right)\right|\right), \text { for } 1 \leq j \leq M \text { and } 1 \leq i \leq N\right\}\right.
$$

\section{Applications}

Step 1. Assume that $u_{1}, u_{2}$, and $u_{3}$ be any three signals received by a digital receiver from any source. Each signal is a triplet of numbers. The first number of triplet represents the truth value, second represents the intermediate value, and the third represents the false value corresponding to each signal. Now, each of these signals is sampled three times. Let $R$ be the given known reference signal. Each signal is compared with the reference signal in order to get the high degree of resemblance with the reference signal $R$. Now, we obtain the matrix $A$ by setting the signals along column and their three times sampling along row. Similarly, we will obtain the matrix $B$.

step 2. Matrices $A$ and $B$ are given by

$$
A=\left[\begin{array}{ccc}
(0.7,0.4,0.5) & (0.6,0.7,1) & (0.8,1,0.7) \\
(0.8,0.5,0.3) & (0.2,0,0.9) & (0.5,0.8,0.4) \\
(0.4,0,0.8) & (0.8,0.4,0.6) & (0,0.3,0.9)
\end{array}\right]
$$

step 3

$$
B=\left[\begin{array}{ccc}
(0.4,0.4,0) & (0.6,0.7,0.4) & (0.1,0.3,0) \\
(0.3,0.7,0.7) & (0.4,0.9,0.4) & (0.1,0.6,0.4) \\
(0.2,0.4,0.5) & (0.4,0.5,0.3) & (0.8,0.5,0.8)
\end{array}\right]
$$

step 4. Now, we will calculate the product of above defined matrices by usual multiplication of matrices. In this multiplication, the truth value of the entry of the first matrix will be multiplied by the truth value of the entry of the second matrix. Similarly, the intermediate and false values of the entries are multiplied.

$$
A B=\left[\begin{array}{ccc}
(0.62,0.69,0.42) & (0.98,0.96,0.45) & (0.77,0.59,0.6) \\
(0.48,0.52,0.83) & (0.76,0.75,0.6) & (0.5,0.55,0.68) \\
(0.4,0.4,0.87) & (0.56,0.51,0.83) & (0.12,0.39,0.96)
\end{array}\right] .
$$

step 5. We calculate CNFSMmDM $[A B]=\left[d_{i 1}\right]$, for all $i=1,2,3$, where $d_{i 1}$ is defined as $d_{i 1}=\min \left\{t_{k 1}\right\}=\min \left\{t_{11}\right.$, $\left.t_{21}, t_{31}\right\}$ for all $k=1,2,3$. 


$$
\begin{aligned}
d_{11} & =\min \left\{t_{k 1}\right\}=\min \left\{t_{11}, t_{21}, t_{31}\right\} \\
& =\min \{(0.62,0.69,0.42),(0.48,0.52,0.83),(0.4,0.4,0.87)\}=(0.4,0.4,0.42), \\
d_{21} & =\min \left\{t_{k 2}\right\}=\min \left\{t_{12}, t_{22}, t_{32}\right\} \\
& =\min \{(0.98,0.96,0.45),(0.76,0.75,0.6),(0.56,0.51,0.83)\}=(0.56,0.51,0.45), \\
d_{31} & =\min \left\{t_{k 3}\right\}=\min \left\{t_{13}, t_{23}, t_{33}\right\} \\
& =\min \{(0.77,0.59,0.6),(0.5,0.55,0.68),(0.12,0.39,0.96)\}=(0.12,0.39,0.6) .
\end{aligned}
$$

We obtain CNFSMmDM as follows:

$$
\text { CNFSMmDM }[A B]=\left[d_{i 1}\right]=\left[\begin{array}{c}
(0.4,0.4,0.42) \\
(0.56,0.51,0.45) \\
(0.12,0.39,0.6)
\end{array}\right] \text {. }
$$

Step 6. Finally, we find out an optimum set on $U$ as follows: $\operatorname{opt}_{M m[A B]}(U)=u_{2}$. So, the signal which is identified as a reference signal is the signal $u_{2}$.

\section{Conclusion}

This paper consists of CNFSM and different types of complex neutrosophic soft matrices with examples. We introduced some new operations on complex neutrosophic fuzzy soft matrices and explore related properties. Further, we constructed a complex neutrosophic soft decisionmaking algorithm with the help of these matrices and used it in signal processing. We hope that our finding will help in enhancing the study on complex neutrosophic soft theory and will open a new direction for applications especially in decision sciences. In future, we will define some new operations on complex neutrosophic fuzzy soft sets and will introduce some new algorithms for signals and other related decision-making in social sciences. Specifically, we will use complex fuzzy sets and complex neutrosophic fuzzy sets in signal processing for modeling of continuous signals.

\section{Conflicts of Interest}

The authors declare that there are no conflicts of interest regarding the publication of this article.

\section{Acknowledgments}

This work was financially supported by the Higher Education Commission of Pakistan (Grant No. 7750/Federal/ NRPU/R\&D/HEC/2017).

\section{References}

[1] L. A. Zadeh, "Fuzzy sets," Information and Control, vol. 8, no. 3, pp. 338-353, 1965.

[2] K. T. Atanassov, "Intuitionistic fuzzy sets," Fuzzy Sets and Systems, vol. 20, no. 1, pp. 87-96, 1986.

[3] D. Molodtsov, "Soft set theory-first results," Computers \& Mathematics with Applications, vol. 37, no. 4-5, pp. 19-31, 1999.
[4] D. A. Molodtsov, "The description of a dependence with the help of soft sets," Journal of Computer and Systems Sciences International, vol. 40, no. 6, pp. 977-984, 2001.

[5] D. A. Molodtsov, The Theory of Soft Sets, URSS Publishers, Moscow, Russia, in Russian, 2004.

[6] D. A. Molodtsov, V. Yu. Leonov, and D. V. Kovkov, "Soft sets technique and its application," Nechetkie Sistemy i Myagkie Vychisleniya, vol. 1, no. 1, pp. 8-39, 2006.

[7] F. Smarandache, "Extension of soft set to hypersoft set, and then to plithogenic hypersoft set," Neutrosophic Sets and Systems, vol. 22, pp. 168-170, 2018.

[8] P. K. Maji, R. Biswas, and A. R. Roy, "Fuzzy soft sets," Journal of Fuzzy Mathematics, vol. 9, no. 3, pp. 589-602, 2001.

[9] P. K. Maji, A. R. Biswas, and A. R. Roy, "An application of soft sets in a decision making problem," Computers \& Mathematics with Applications, vol. 44, no. 8-9, pp. 1077-1083, 2002.

[10] N. Cagman and S. Enginolu, "Soft matrix theory and its decision making," Computers and Mathematics with Applications, vol. 59, pp. 3308-3314, 2010.

[11] F. Smarandache, A Unifying Field in Logics Neutrosophy: Neutrosophic Probability, Set and Logic, American Research Press, Rehoboth, DE, USA, 1999.

[12] F. Smarandache, Plithogeny, Plithogenic Set, Logic, Probability, and Statistics, Pons Publishing House, Brussels, Belgium, 2017.

[13] N. A. Nabeeh, F. Smarandache, M. Abdel-Basset, H. A. ElGhareeb, and A. Aboelfetouh, "An integrated neutrosophicTOPSIS approach and its application to personnel selection: a new trend in brain processing and analysis," IEEE Access, vol. 7, pp. 29734-29744, 2019.

[14] M. Abdel-Basset, M. Saleh, A. Gamal, and F. Smarandache, "An approach of TOPSIS technique for developing supplier selection with group decision making under type-2 neutrosophic number," Applied Soft Computing, vol. 77, pp. 438-452, 2019.

[15] M. Abdel-Baset, V. Chang, A. Gamal, and F. Smarandache, "An integrated neutrosophic ANP and VIKOR method for achieving sustainable supplier selection: a case study in importing field," Computers in Industry, vol. 106, pp. 94-110, 2019.

[16] M. Ali and F. Smarandache, "Complex neutrosophic set," Neural Computing \& Applications, vol. 28, no. 7, pp. 18171834, 2016.

[17] Y. Yang and C. Ji, "Fuzzy soft matrices and their applications," Artificial Intelligence and Computational Intelligence, vol. 7002, pp. 618-627, 2011.

[18] D. Ramot, R. Milo, M. Friedman, and A. Kandel, "Complex fuzzy sets,” IEEE Transactions on Fuzzy Systems, vol. 10, no. 2, pp. 171-186, 2002.

[19] A. Alkouri and A. Salleh, "Complex intuitionistic fuzzy sets," in Proceedings of the International Conference on Fundamental and Applied Sciences, AIP Conference Proceedings, vol. 1482, pp. 464-470, Kuala Lumpur, Malaysia, June 2012. 
[20] K.-Y. Cai, " $\delta$-Equalities of fuzzy sets," Fuzzy Sets and Systems, vol. 76, no. 1, pp. 97-112, 1995.

[21] Y. K. Cai, "Robustness of fuzzy reasoning and $\delta$-equalities of fuzzy sets," IEEE Transactions on Fuzzy Systems, vol. 9, no. 5, pp. 738-750, 2001.

[22] G. Zhang, K.-Y. Cai, J. Lu, and J. Lu, "Operation properties and $\delta$-equalities of complex fuzzy sets," International Journal of Approximate Reasoning, vol. 50, no. 8, pp. 1227-1249, 2009. 